\title{
Viewpoint
}

\section{Vascular anatomy in gastrointestinal inflammation}

\author{
Andrew Anthony
}

Over the past 10 years, much of the work on the development of new treatments for inflammatory and ulcerative disorders of the gut, such as inflammatory bowel disease, has focused on the cellular and molecular aspects of the inflammatory process. This is a direct result of the identification of the numerous inflammatory mediators involved and application of powerful molecular biological techniques. Emphasis upon control of acid secretion, followed rapidly by the discovery of Helicobacter pylori, has meant that knowledge acquired from such basic research on the inflammatory process has bypassed peptic ulcer disease, but it has potential application in the development of novel inflammatory inhibitors for inflammatory bowel disease. The use of such biological treatment for inflammatory bowel disease (for example, anti-TNF antibodies) has produced some promising preliminary results and encouraged more extensive clinical trials. ${ }^{12}$

Pathological inflammation, defined as the humoral and cellular response to tissue injury, may have been overplayed by those wishing to evaluate new treatment options. One way of looking at inflammation is to think of it as representing the blaze and smoke of a fire; hitherto, much of the research effort has focused on developing agents that attempt to dampen the flame. However, some have commented on the naivity of trying to control gastrointestinal inflammation by targeting a solitary cytokine, despite moderately encouraging early results. ${ }^{34}$ Singling out one cytokine may simply leave others to take over. ${ }^{4}$ It can be anticipated that when the initiating factors (that is, the primary causes) involved in igniting the flame are identified, research efforts will invariably shift to other forms of treatment.

During my research work on inflammatory bowel disease over the past seven years, it has become apparent there are some fundamental and well documented idiosyncrasies of inflammatory and ulcerative diseases of the gut that have hitherto been overlooked and not considered seriously by most clinical and experimental gastroenterologists. One such idiosyncrasy I would like to expand in this article is the association between the specific anatomical distribution of tissue injury and the vascular anatomy of the gut. On closer examination, it seems that some of the common disorders of the gut - including peptic ulcer disease, inflammatory bowel disease, and anal fissures - show distinct anatomical focality, the distribution of which can be resolved to the vascular anatomy of the gut wall. More specifically, the defined regions of the bowel wall that are prone to developing lesions happen to be poorly vascularised, so that for each of the conditions mentioned above, a vascular hypothesis that promotes a notion of ischaemic injury to the mucosa can be applied.

Well specified areas of the bowel that are prone to both ischaemia and ulceration in peptic ulcer disease, inflammatory bowel disease, and anal fissures will be defined and discussed. There will be particular reference to studies, some of them performed by our group at the Royal Free Hospital, that have attempted to associate the predilection of these sites to mucosal damage with deficiency of the blood supply, as determined by detailed examination of the vascular anatomy.

\section{Peptic ulcer disease}

All medical students are taught that benign peptic ulcers of the stomach occur mainly along the lesser curvature of the antrum. This fundamental observation itself indicates that some anatomical feature of this site must be involved in the pathogenic mechanisms of gastric ulcer formation. In studies that examined the vascular anatomy of the human stomach and duodenum, Piasecki identified end artery systems that were considered to play a crucial role in the peptic ulcer disease phenotype. ${ }^{5}$ By studying carbon ink perfused gastrectomy specimens he found, within the first part of the duodenum and lesser curvature of the antrum, end arteries that arose from the extramural blood supply without connecting with the submucosal plexus of vessels. The distribution of 47 such vessels found in 10 non-ulcerated human specimens corresponded exactly to the preferred sites of chronic gastroduodenal peptic ulcer disease. ${ }^{56} \mathrm{He}$ hypothesised that in individuals possessing such end arteries, initiating mechanisms that might induce prolonged vasospasm or muscular contraction during periods of stress would cause mucosal ischaemia and necrosis. The observation that not all stomachs had such end arteries but had instead a continuous collateral circulation within the submucosa could explain, in part, why some individuals do not develop peptic ulcer disease despite having raised gastric acid secretion and $H$ pylori infection. This hypothesis was later
Accepted for publication 4 February 1999 
supported by in vivo animal work, where ligation of supplying arteries and muscular compression of blood vessels was found to cause reductions in mucosal blood flow and mucosal ulceration. ${ }^{78} \mathrm{~A}$ role for vascular and non-vascular smooth muscle in human peptic ulcer disease is supported by pharmacological studies where spasmolytic agents and vasodilators inhibit experimental gastric lesions, the pathological and anatomical nature of which strongly resembles human peptic ulcers. ${ }^{9}{ }^{10}$

Acid suppression treatment is currently a safe means of treating chronic peptic ulcers but if, in the future, long term acid suppression is found to be unacceptably harmful, then alternative approaches to treatment will need to be considered. Appropriate selective spasmolytics or vasodilators that are able to overcome mucosal ischaemia in susceptible sites of the stomach and duodenum might fulfil such a role.

By stimulating inflammatory cytokine release within the gastroduodenal mucosa, $H p y$ lori is now considered to play a primary role in peptic ulcer disease. Recently, Kalia et al found that extracts from human $H$ pylori cultures applied to the gastric mucosa of mice caused early microvascular leakage and platelet aggregation before neutrophil recruitment. ${ }^{11}$ Whether $H$ pylori infection causes injury to the mucosal microvasculature, or indeed to the deeper larger vessels, remains to be seen.

\section{Inflammatory bowel disease}

CROHN'S DISEASE

A recognised but until most recently unexplained feature of Crohn's disease ulcers in the ileum was their propensity to occur along the mesenteric margin of the bowel wall (fig 1). ${ }^{12-18}$ In this chronic disorder, there is evidence that the underlying pathogenic mechanism involves inflammation of supplying blood vessels. This hypothesis was proposed by Wakefield et al who, on the basis of immunohistological evidence which associated granulomata with the vasculature, suggested that ischaemic mucosal injury occurs as a result of occlusive granulomatous vasculitis. ${ }^{19}{ }^{20}$ We recently examined the possibility that the mesenteric margin was more susceptible than the antimesenteric margin to ischaemia and would be prone to ulceration if blood flow to this site was relatively compromised for reasons that might involve the vasculature. Upon examination of the arterial blood supply of necropsy specimens of normal ileum, we identified small mural blood vessels (short vessels) that selectively supplied the submucosal plexus and mucosa of the mesenteric margin and had virtually no communication with larger feeding vessels (long vessels) that supplied the mucosa of the anti-mesenteric margin - that is, the former were end arteries (fig 2). Equivalent short vessels in the jejunum, however, were not end arteries as they had extensive communication with the submucosal plexus of vessels arising from long vessels. We suggested that an occlusive vascular event occurring at any site along ileal short vessels would cause ischaemic ulceration to the mucosa along the mesenteric

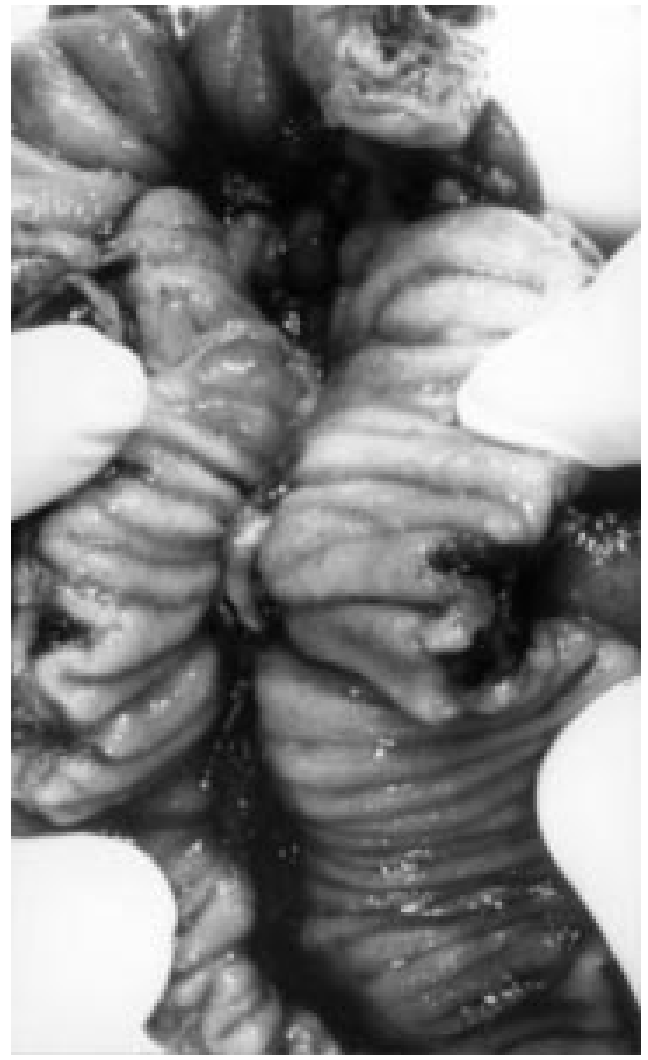

Figure 1 Surgical specimen of ileal Crohn's disease showing a longitudinal ulcer along the mesenteric margin. The adjacent mucosa has a normal appearance.

margin and could reasonably explain the preferential ulceration of the mesenteric margin in Crohn's disease of the terminal ileum.

When we examined histologically the association of granulomas with the extramural vasculature in 12 Crohn's disease resection specimens, 10 cases (six ileal; four colonic) showed multifocal granulomatous vasculitis of arteries and veins in which there was clear destructive and in some cases occlusive vasculopathy (unpublished observations). We carefully looked at the path of extramural vessels in longitudinal rather than transverse section so that we could examine the vessels for an appreciable length; this was best at highlighting the multifocal nature of the vascular pathology. Although others doubt a role for the vasculature and propose a primary role for the lymphatics, ${ }^{21}$ we are convinced that granulomatous vasculitis is a common pathological feature of Crohn's disease and plays a role in an ischaemic pathogenesis in this disorder.

Along the same lines, Funayama et al also identified large anastomotic vessels at the antimesenteric margin of the small intestine that were less abundant along the mesenteric margin ${ }^{17}$; they proposed that in Crohn's disease the mesenteric margin was more susceptible to episodes of ischaemia and hence more liable to ulceration.

During our studies of an experimental model of inflammatory bowel disease (indomethacin jejunitis in the rat) we noted that most longitudinal jejunal ulcers had a remarkable resemblance to Crohn's disease ulcers in that they 


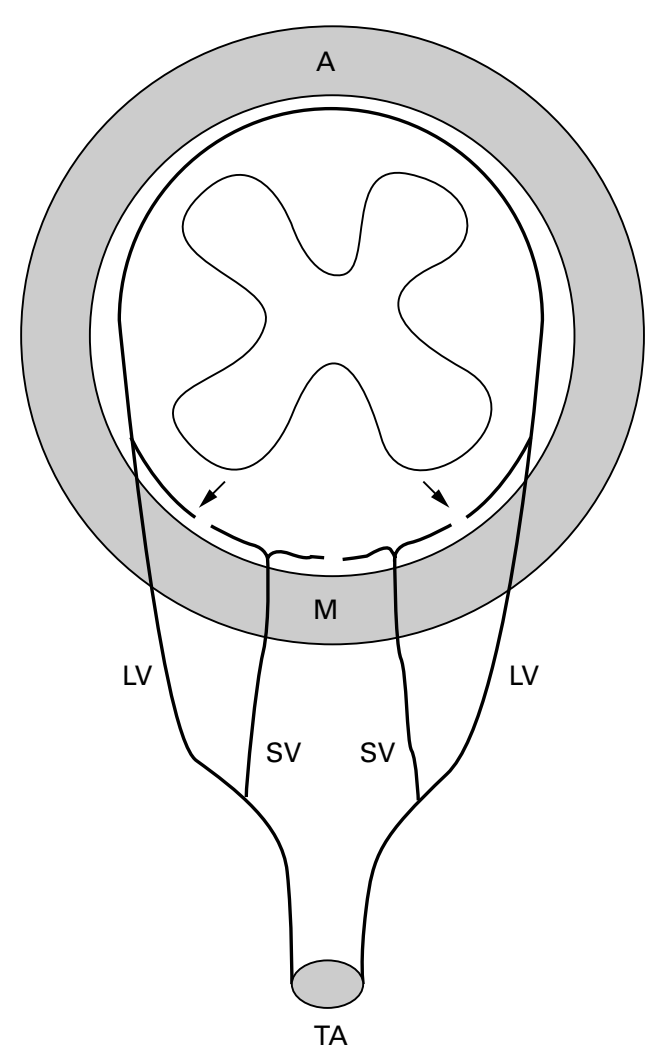

Figure 2 Schematic diagram of the blood supply to the human ileum. The mesenteric margin (M) is supplied by short vessels $(S V)$, while the anti-mesenteric margin $(A)$ is supplied by longer vessels ( $L V)$. There are virtually no connections (at the points denoted by arrows) between the submucosal plexuses derived from SV and LV. TA, terminal arcade vessel.

also had a preference for the mesenteric margin (fig 3). As in the human ileum, we found the ulcer susceptible mesenteric margin to be supplied by short end arteries. ${ }^{22}$ We proposed that indomethacin induced ischaemia to the mesenteric margin either by vasoconstriction or by squeezing short vessels by spasm of the muscularis propria. Evidence for a role of vascular or non-vascular smooth muscle in this animal model is provided by the finding that the spasmolytic/vasodilator CL316243 (a $\beta_{3}$ adrenoceptor agonist) and atropine (a spasmolytic muscarinic receptor antagonist) are both inhibitors of indomethacin induced small bowel ulceration in the rat. ${ }^{2324}$

\section{ULCERATIVE COLITIS}

Hamilton et al proposed that the sharp demarcation between normal and diseased bowel in ulcerative colitis could not be explained by current hypotheses such as a luminal antigen, abnormal mucus production, or an autoimmune response against some epithelial antigen. ${ }^{25}$ Using in vitro angiography, they found that the extent of colitis was defined by the segment of the colon supplied by the marginal artery (Arteria marginalis coll), the most proximal branch of the inferior mesenteric artery. They suggested that the extent of disease might be explained in terms of the embryological origin of the inferior mesenteric artery and its associated autonomic nerve supply.

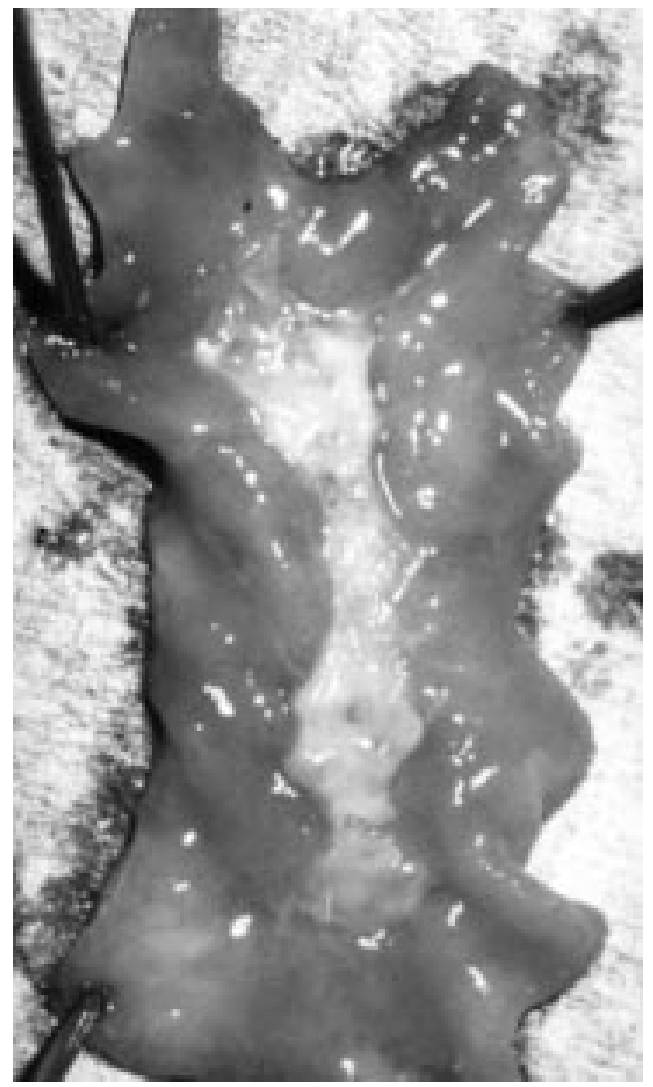

Figure 3 Mesenteric marginal ulceration induced by indomethacin in the rat jejunum (reproduced with permission).

\section{Anal fissures}

Chronic anal fissures are thought to arise as a consequence of increased anal sphincter tone which impedes blood flow to the anal mucosa. ${ }^{26}$ The fissures are therefore considered to be ischaemic ulcers of the anal canal. In about $85 \%$ of cases the fissures are localised to the posterior commissure midline of the anal canal. Examining the vascular anatomy of the anal canal using postmortem angiography, Klosterhalfen et al found that the posterior commissure was less perfused than other areas of the anal canal, and rectal vessels within this area rarely formed anastamoses. ${ }^{27}$ They also considered that the vertical passage of arteries through the anal sphincters would contribute to ischaemia of the anal canal during strong contractions. The ischaemic nature of anal fissures was studied further by Schouten et al, who showed that anal resting pressure was higher (and anodermal blood flow lower) in subjects with anal fissure compared with control subjects. ${ }^{28}$ In the same study, sphincterotomy healed 24 of 27 patients with fissures within six weeks; there was also a decrease in resting pressure which was accompanied by a rise in blood flow following the procedure. The investigators concluded that sphincterotomy improves anodermal blood flow, resulting in fissure healing.

A major complication with both sphincterotomy and anal dilatation is that a significant proportion of patients develop incontinence. The development of a non-surgical treatment of raised anal pressure would therefore be 
useful. Recently, nitric oxide has been shown to be an inhibitor of the internal anal sphincter ${ }^{29}$; the nitric oxide donor, glyceryl trinitrate, caused a fall in maximum resting anal pressure after topical application to the anal mucosa and healed $68 \%$ of anal fissures in an eight week double blind, placebo controlled trial, compared with an $8 \%$ healing rate in the placebo group. ${ }^{30}$ However, glyceryl trinitrate often causes headaches which might limit its therapeutic use, so other agents with similar pharmacological properties should be sought. The calcium channel blocker diltiazem has recently been shown to lower resting anal tone and is currently being evaluated for use in the treatment of anal fissures. ${ }^{31}$

\section{Comment}

For inflammatory bowel disease and peptic ulcer disease a role for the vasculature in pathogenic mechanisms has hitherto been largely overlooked. For compelling anatomical reasons a case has been made for, first, a primary role of the vasculature in peptic ulcer disease, inflammatory bowel disease, and anal fissures, and second, treatment of these disorders of the gastrointestinal tract by classes of agents that improve blood flow to regions of the gut wall susceptible to ischaemia.

1 van Deventer SJH, Camoglio L. Monoclonal antibody therapy of inflammatory bowel disease. Aliment Pharmacol Ther 1996;10(suppl 2):107-11.

2 Sands BE. Biologic therapy for inflammatory bowel disease. Inflam Bowel Dis 1997;3:95-113.

3 O'Farrelly C. Just how inflamed is the normal gut? Gut 1998;42:603-4.

4 Bonn D. Tackling the real culprits in Crohn's disease. Lancet 1998;351:1710

5 Piasecki C. Blood supply to the human gastroduodenal mucosa with special reference to ulcer bearing areas. 7 Anat 1974;118:295-335.

6 Piasecki C. Blood supply to the human gastroduodenal mucosa with special reference to the ulcer bearing areas. $B$ 7 Surg 1971;58:660-2.

7 Piasecki C, Thrasivoulou C, Rahim A. Ulcers produced by ligation of individual gastric mucosal arteries in the guinea pig. Gastroenterology 1989;97:1121-9.

8 Piasecki C, Thrasivoulou C. Spasm of gastric muscularis mucosae might play a key role in causing focal ischaemia and necrosis; an experimental study in guinea pigs. Dig Di Sci 1993;38:1183-9.

9 Takeuchi K, Okada M, Ebara S, et al. Increased microvascular permeability and lesion formation during gastric hypermotility caused by indomethacin and 2-deoxy-D-glucose in the rat. f Clin Gastroenterol 1990;12(suppl 1):S76-84.
10 Kuratani K, Kodama H, Yamaguchi I. Enhancement of mucosal blood flow by beta-3-adrenergic agonists prevents macol Exp Ther 1994;270:559-65.

11 Kalia N, Jacob S, Brown NJ, et al. Studies on the gastric mucosal microcirculation. 2. Helicobacter pylori water soluble extracts induce platelet aggregation in the gastric mucosal microcirculation in vivo. Gut 1997;41:748-56.

12 Crohn BB, Ginsberg L, Oppenheimer G. Regional ileitis. A pathological and clinical entity. $\mathcal{F} A M A$ 1932;99:1323-9.

13 van Patter WN, Bargen JA, Dockerty MB, et al. Regional enteritis. Gastroenterology 1954;26:347-450.

14 Warren S, Sommers. Pathology of regional ileitis and ulcerative colitis. $\mathcal{F A M A}$ 1954;154:189-93.

15 Brahme F, Lindström C. A comparative radiographic and pathological study of intestinal architecture in Crohn's disease and in ulcerative colitis. Gut 1970;11:928-40.

16 Yagamata S. Crohn's disease in Japan: report of the Japanese committee for Crohn's disease. In: Lee ECG, ed. Crohn's workshop-a global assessment of Crohn's disease. London: HMSO, 1981 .

17 Funayama Y, Sasaki I, Naito Y, et al. Micro-angiographic studies of intestinal vasoarchitecture with special reference to ulcerogenesis in Crohn's disease. 7 fpn Soc Coloproctol 1987;40:839-44.

18 Anthony A, Dhillon AP, Pounder RE, et al. Ulceration of the leum in Crohn's disease: correlation with vascular anatomy. F Clin Pathol 1997;50:1013-17.

19 Wakefield AJ, Sankey EA, Dhillon AP, et al. Granulomatous vasculitis in Crohn's disease. Gastroenterology 1991;100: 1279-87.

20 Wakefield AJ, Dhillon AP, Rowles PM, et al. Pathogenesis of Crohn's disease: multifocal gastrointestinal infarction. Lancet 1989; ii:1057-62.

21 Mooney EE, Walker J, Hourihane DOB. Relation of granulomas to lymphatic vessels in Crohn's disease. 7 Clin Pathol 1995;48:335-8

22 Anthony A, Dhillon AP, Pounder RE, et al. Vascular anatomy defines sites of indomethacin-induced jejunal ulceration along the mesenteric margin. Gut 1997;41:76370 .

23 Anthony A, Bahl AK, Dhillon AP, et al. The beta ${ }_{3}^{-}$ adrenoceptor agonist CL3162343 prevents indomethacininduced jejunal ulceration in the rat by reversing early villous shortening. F Pathol 1996;179:340-6.

24 Karadi O, Abdel-Salam OME, Bodis B, et al. Preventive effect of atropine on indomethacin-induced gastrointestinal mucosal and vascular damage in rats. Pharmacology 1996;52:46-55.

25 Hamilton MI, Dick R, Crawford L, et al. Is proximal demarcation of ulcerative colitis determined by the territory of the inferior mesenteric artery? Lancet 1995;345:688-90.

26 Gibbons CP, Read NW. Anal hypertonia in fissures: cause or effect? Br F Surg 1986;73:443-5.

27 Klosterhalfen B, Vogel P, Rixen H, et al. Topography of the inferior rectal artery: a possible cause of chronic primary anal fissure. Dis Colon Rectum 1989;32:43-5.

28 Schouten WR, Briel JW, Auwerda JJA, et al. Ischaemic nature of anal fissure. BrF Surg 1996;83:63-5.

29 O'Kelly TJ. Nerves that say NO: a new perspective on the human rectoanal inhibitory reflex. Ann R Coll Surg Eng 1996;78:31-8

30 Lund JN, Scholefield JH. A randomised, prospective placebo-controlled trial of glyceryl trinitrate ointment in treatment of anal fissure. Lancet 1997;349:11-14.

31 Carapeti EA, Kamm MA, Evans BK, et al. Diltiazem lowers resting anal sphincter pressure: a potential low side-effect alternative to glyceryl trinitrate (GTN) for fissures [abstract]. Gastroenterology 1998;114:A7. 